\title{
Determinación del costo de producción de papa nativa, Cusco
}

\section{Determination of the cost of production of native potatoes, Cusco}

\section{Determinação do custo de produção de batata nativa, Cusco}

\author{
Yoni Sivincha Domínguez \\ yoni.sivincha@upeu.edu.pe \\ https://orcid.org/0000-0001-4946-6878 \\ Universidad Peruana Unión, Perú \\ Jesús Mariano Gómez Miguel \\ jgomezmiguel1@gmail.com \\ (iD https://orcid.org/0000-0003-0706-072X \\ Universidad Nacional Daniel Alcides \\ Carrión, Perú
}

\author{
Pío Augusto Champi Huanca \\ piochampi@upeu.edu.pe \\ iD https://orcid.org/0000-0001-6725-0926 \\ Hebert Carlos Castillo Paredes \\ ccastillop@undac.edu.pe \\ iD https://orcid.org/0000-0001-7443-5283 \\ Universidad Nacional Daniel Alcides \\ Carrión, Perú
}

Recibido: 18 de junio de 2021

Aceptado: 30 de diciembre de 2021

\section{Resumen}

Actualmente en la comunidad de Pumapaqcha, la producción anual de papa supera las treinta toneladas; es el principal ingreso económico de ochenta familias aproximadamente; no le dan la suficiente importancia al costo de producción: herramienta para la planeación y toma de decisiones. Dejan al margen algunos desembolsos importantes que afectan el costo del producto, generando bajos niveles de rentabilidad, bajas ganancias, devaluación de la producción, menoscabando el desarrollo económico familiar. La presente investigación tiene el objetivo principal de analizar los costos de producción de papa nativa; el tipo investigación es evaluativa; se utilizó el sistema tradicional de asignación de costos. En sus resultados se registra que el costo de producción por kilo es S/ 1.10 soles; en una producción de 0.25 hectáreas, el costo de producción total es $\mathrm{S} / 2751.00$ soles. El precio de venta de la papa nativa es $\mathrm{S} / 1.00$, provocando un margen bruto de rentabilidad de $-10 \%$. En conclusión, existe la necesidad de optimizar los costos de producción, en los procesos de preparación de la tierra y la siembra de la producción de papa nativa. 
Palabras clave: Análisis de costos, rentabilidad, sistema tradicional de asignación de costos.

\begin{abstract}
Currently in the Pumapaqcha community, annual potato production exceeds thirty tons; It is the main economic income of approximately eighty families; they do not give enough importance to the cost of production: a tool for planning and decision making. They leave aside some important disbursements that affect the cost of the product, generating low levels of profitability, low profits, devaluation of production, undermining family economic development. The present investigation has the main objective of analyzing the production costs of native potatoes; the research type is evaluative; the traditional cost allocation system was used. Its results show that the cost of production per kilo is $\mathrm{S} / 1.10$ soles; In a production of 0.25 hectares, the total production cost is S / 2751.00 soles. The sale price of native potatoes is $\mathrm{S} / 1.00$, causing a gross profit margin of $-10 \%$. In conclusion, there is a need to optimize production costs in land preparation processes and planting of native potato production.
\end{abstract}

Keywords: Cost analysis, profitability, traditional cost allocation system.

\title{
Resumo
}

Atualmente na comunidade Pumapaqcha, a produção anual de batata excede trinta toneladas; é a principal renda econômica de aproximadamente oitenta famílias; não dão importância suficiente ao custo de produção: uma ferramenta de planejamento e tomada de decisão. Deixam de lado alguns desembolsos importantes que afetam o custo do produto, gerando baixos níveis de lucratividade, baixos lucros, desvalorização da produção, prejudicando o desenvolvimento econômico familiar. A presente investigação tem como objetivo principal analisar os custos de produção da batata nativa; o tipo de pesquisa é avaliativo; o sistema tradicional de alocação de custos foi usado. Seus resultados mostram que o custo de produção por quilo é de $\mathrm{S} /$ 1,10 soles; Em uma produção de 0,25 hectares, o custo total de produção é de S / 2.751,00 soles. O preço de venda da batata nativa é de $\mathrm{S} / 1,00$, resultando em uma margem de lucro bruto de $-10 \%$. Conclui-se que existe a necessidade de otimizar os custos de produção nos processos de preparo do solo e plantio da produção de batata nativa. 
Palavras-chave: Análise de custos, lucratividade, sistema tradicional de alocação de custos.

\section{Introducción}

Según los datos aportados por la Organización de Alimentos y Agricultura de las Naciones Unidas (FAO), el incremento de la producción de papa a nivel mundial ha sido generado, en gran medida, por las nuevas variedades y la aplicación de nuevas tecnologías de producción (FAO, 2003). Estos cambios en las tecnologías de producción han producido, a su vez, cambios en el aspecto contable y en el cálculo del costo de producción; cualquier modificación en los métodos de producción afecta directamente el costo del producto. Han surgido diferentes metodologías, para determinar correctamente el costo de los productos, aplicables fácilmente en el sector agrícola.

Tradicionalmente, el costo de producción está compuesto por los materiales directos, la mano de obra directa y los costos indirectos de fabricación. Estos últimos son, según bases de distribución, relacionados con el nivel de producción. Con el devenir del tiempo han surgido otras formas de asignación de los costos indirectos de fabricación; a los productos se aplican de manera más natural los procesos agrícolas (Molina De Paredes y Contreras, 2005).

En la actualidad y en la comunidad de Pumapaqcha, la producción anual de papa supera las treinta toneladas, constituyéndose en la fuente principal de ingreso económico, para ochenta familias aproximadamente. A pesar de esto, los productores no le dan suficiente importancia al cálculo del costo de producción: una herramienta para la planeación y toma de decisiones, ni siquiera es considerado el elemento fundamental, para calcular su rentabilidad con precisión. Además, dejan al margen algunos desembolsos importantes, los cuales afectan el costo del producto (Manjarrés, 2003).

Para mejorar la rentabilidad de la producción de papa se deben aplicar estrategias, para el aumento de la superficie sembrada, el incremento de la productividad, la introducción de mejoras tecnológicas y/o el mejoramiento de su capacidad gerencial. Sin embargo, antes de la aplicación de cualquier estrategia es necesario poseer un adecuado sistema de costos que incluya todos los elementos que intervienen en cada una de las etapas del proceso productivo (Molina de Paredes y Contreras, 2005). 
Molina de Paredes y Contreras (2005) realizaron un estudio planteando el objetivo: analizar el cálculo del costo de producción de papa en Venezuela. La investigación fue de tipo evaluativa, con una revisión de documentos y la aplicación de instrumentos: la guía de observación; se obtuvo los resultados mediante cálculos del costo de cada uno de los rubros sembrados, incluyendo la papa en sus diferentes variedades, lo hacen empíricamente, aparentemente se parece a los costos de absorción. La acumulación se realiza por hectárea sembrada, para cada variedad de papa por separado y para cada ciclo de producción. Inclusive obvian ciertos costos para una determinación final del costo total.

Segura (2014), en España, planteó el objetivo: mejorar las condiciones económicas y sociales de las comunidades agrícolas, así como caracterizar los eslabones de la cadena de valor de la papa nativa y sus derivados en la provincia de Jauja del Perú, usando un análisis DAFO, verificando las debilidades y fortalezas de cada eslabón de valor. Se obtuvieron recomendaciones para mejorar las condiciones de venta y mejores precios de sus productos, lo que mejora las condiciones de vida de los pobladores de la comunidad.

Chani y Pfuro (2015) llegaron a la conclusión de que las zonas productoras de papas nativas son mayores que años anteriores, debido a un incremento de la demanda de este producto; los consumidores ahora prefieren consumir alimentos sanos y naturales, que les ayuden a cuidar y mantener su salud.

Minaya (2016) realizó una investigación en Perú; planteó el objetivo: “Comparar efectos de las variables que determinan los niveles de rentabilidad en la producción de papa blanca comercial en las regiones de Huánuco y Lima”. Llegó a la conclusión de que las principales fuentes de riesgo, para ambas regiones, son el precio en la misma chacra, cuyos dueños cobran por kilo de papa blanca comercial, así como la productividad o rendimiento por hectárea, y en menor medida los costos de pesticidas.

El presente artículo de investigación beneficiará a todos los productores de papa nativa de la provincia de Paucartambo; porque en toda actividad económica, la agricultura cultiva y comercializa un determinado producto, depende en gran parte del manejo de los costos en el cultivo; un buen método de costeo con un control ofrecerá información actualizada permitiendo tomar decisiones más acertadas. Se obtienen así mejores ganancias, mediante un control de costos adecuado. Por todo lo mencionado, el objetivo de esta investigación fue: determinar los costos de producción de papa nativa de los 
productores de la comunidad de Pumapaqcha distrito de Colquepata de la provincia de Paucartambo en la región de Cusco

\section{Metodología}

\section{Población}

La población de estudio está constituida por 80 comuneros productores de papa nativa, residentes en la comunidad campesina de Pumapaqcha, Colquepata, Paucartambo.

\section{Muestra}

Para la muestra se consideró 0.25 hectáreas que equivale a una producción de 2,500 kilos de papa nativa, porque es la cantidad promedio que posee cada miembro de la comunidad, por causa de la minifundización, el cual consiste en la repartición hereditaria de los lotes de los propietarios.

\section{Técnicas de recolección de datos}

\section{Análisis documental}

La recolección de los datos se realizó a través de registros, informes, libros, reuniones y entrevistas con los miembros de la comunidad, que se utilizaron para el análisis de la rentabilidad (Tamayo y Silva, 2010)

\section{Diseño metodológico}

La naturaleza de este artículo de investigación corresponde al enfoque cuantitativo, nivel descriptivo, como menciona Niño (2011), quien comenta sobre este tipo de investigación: "Su propósito es describir la realidad objeto de estudio, un aspecto de ella, sus partes, sus clases, sus categorías o las relaciones que se pueden establecer entre varios objetos, con el fin de esclarecer una verdad" (p. 33). Asimismo, el diseño será transeccional o transversal, debido a que el registro de datos se realiza en un tiempo determinado (Hernández y Mendoza, 2018).

\section{Técnicas para el procesamiento de la información}

El procesamiento de la información se realizó mediante diagramas de flujo, que permitieron la descripción de dicho proceso. A continuación, en la Figura 1 se muestra el proceso seguido en el estudio de los costos de producción. 


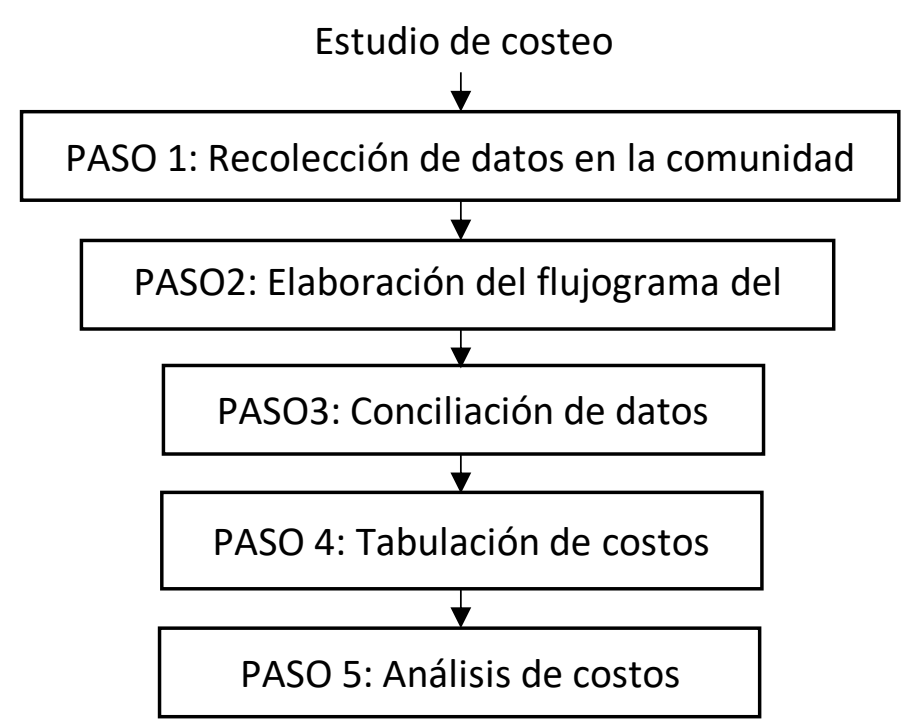

Figura 1 Diagrama de flujo del proceso de costeo de papa nativa.

\section{PASO 1: Recolección de datos en la comunidad}

En este primer paso, se programaron reuniones con los productores de papa nativa de la comunidad, se dio a conocer los objetivos de la investigación y la necesidad de su aplicación en la producción de papa nativa; para la recolección de los datos se empleó una encuesta.

\section{PASO 2: Elaboración de flujograma de proceso de producción de papa nativa}

Para conocer todos los elementos que intervienen en los costos de la producción de papa nativa, fue necesario conocer cada etapa de proceso por medio de entrevistas y encuestas realizadas a los miembros de la comunidad, luego elaborar el diagrama de flujo, así como se muestra en la figura 2. 


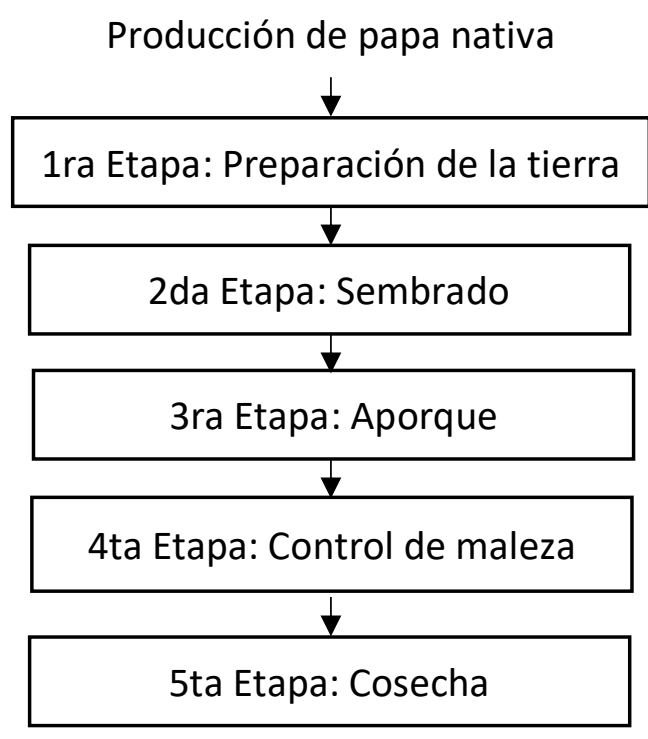

Figura 2. Diagrama de flujo de la producción de papa nativa.

\section{PASO 3: Conciliación de datos que intervienen en la producción}

A continuación, se muestra los datos de los elementos que intervienen en el proceso de cultivo de papa nativa; se consideran los materiales, materia prima, mano de obra y gastos administrativos.

Tabla 1

Conciliación de todos los elementos que intervienen en el proceso de producción de 2500 kilos de papa nativa.

\begin{tabular}{lllll}
\hline 1era Etapa: & 2da Etapa: & 3era Etapa: & 4ta Etapa: & 5ta Etapa: \\
\hline Preparación de la tierra & Siembra & Aporque & Control de maleza & Cosecha \\
\hline Mano de obra & Semilla & Mano de obra & Peón & Peón de recojo \\
Yuntas & Abono & Comida & Comida & Comida \\
Herramientas & Peón guano & Chicha & Chicha & Chicha \\
Comida & Peón siembra & Hojas de coca & Hojas de coca & Hojas de coca \\
Chicha & Comida & Cañazo & Cañazo & Cañazo \\
Hojas de coca & Chicha & Cocinera & Cocinera & Cocinera \\
Cañazo & Hojas de coca & & & Costales \\
Pago a la cocinera & Cañazo & & & Transporte \\
Pago a la tierra & Cocinera & & & \\
\hline
\end{tabular}

\section{PASO 4: Tabulación de costos}

Para la tabulación de costos se elaboraron tablas por cada etapa. En la tabla 2, se muestran todos los elementos que interviene en el proceso de preparación de la tierra, con su respectivo costo unitario, calculados sobre la base de una producción de 0.25 hectáreas (2500 m2); las mismas equivalen a una producción de 2500 kilos de papa nativa. 
Tabla 2

Tabulación del costo real en el proceso de preparación de la tierra

\begin{tabular}{|c|c|c|c|c|}
\hline 1ra etapa: & Preparació & tierra & & \\
\hline Descripción & Medida & Cantidad & Costo Unitario & Importe \\
\hline Mano de obra & Jornal & 12 & $\mathrm{~S} / 30.00$ & $\mathrm{~S} / 360.00$ \\
\hline Yuntas & Unidades & 2 & $\mathrm{~S} / 55.00$ & $\mathrm{~S} / 110.00$ \\
\hline Herramientas & Unidades & 15 & $\mathrm{~S} / 22.00$ & $\mathrm{~S} / 330.00$ \\
\hline Comida & Por peón & 12 & $\mathrm{~S} / 5.00$ & $\mathrm{~S} / 60.00$ \\
\hline Chicha & Porongo & 1 & $\mathrm{~S} / 20.00$ & $\mathrm{~S} / 20.00$ \\
\hline Hojas de coca & Libra & 1 & $\mathrm{~S} / 12.00$ & $\mathrm{~S} / 12.00$ \\
\hline Cañazo & Botella & 1 & $\mathrm{~S} / 14.00$ & $\mathrm{~S} / 14.00$ \\
\hline Pago a la cocinera & Jornal & 1 & $\mathrm{~S} / 20.00$ & $\mathrm{~S} / 20.00$ \\
\hline Pago a la tierra & Jornal & 1 & $\mathrm{~S} / 30.00$ & $\mathrm{~S} / 30.00$ \\
\hline Total (a) & & & & $\mathrm{S} / 956.00$ \\
\hline
\end{tabular}

En la tabla 3, se muestra todos los elementos que interviene en el proceso de siembra de la papa nativa con su respectivo costo unitario.

Tabla 3

Tabulación del costo real en el proceso siembra de papa nativa

\begin{tabular}{lllll}
\hline 2da etapa: & Proceso de siembra & & & \\
\hline Descripción & Medida & Cantidad & Costo Unitario & Importe \\
\hline Semilla & Arroba & 6 & $\mathrm{~S} / 20.00$ & $\mathrm{~S} / 72.00$ \\
Abono & Saco & 6 & $\mathrm{~S} / 10.00$ & $\mathrm{~S} / 60.00$ \\
Peón para abonar & Jornal & 8 & $\mathrm{~S} / 30.00$ & $\mathrm{~S} / 240.00$ \\
Peón para siembra & Jornal & 8 & $\mathrm{~S} / 30.00$ & $\mathrm{~S} / 240.00$ \\
Comida & Por peón & 16 & $\mathrm{~S} / 5.00$ & $\mathrm{~S} / 80.00$ \\
Chicha & Porongo & 1 & $\mathrm{~S} / 20.00$ & $\mathrm{~S} / 20.00$ \\
Hojas de coca & Libra & 1 & $\mathrm{~S} / 12.00$ & $\mathrm{~S} / 12.00$ \\
Cañazo & Botella & 1 & $\mathrm{~S} / 14.00$ & $\mathrm{~S} / 14.00$ \\
Pago a la cocinera & & 1 & $\mathrm{~S} / 20.00$ & $\mathrm{~S} / 20.00$ \\
\hline Total (b) & & & $\mathrm{S} / 758.00$ \\
\hline
\end{tabular}

En la tabla 4, se muestra todos los elementos que interviene en el proceso de aporque de los terrenos de cultivo de la papa nativa, con su respectivo costo unitario. 
Tabla 4

Tabulación del costo real del proceso de aporque de los terrenos

\begin{tabular}{lllll}
\hline 3ra etapa: & \multicolumn{3}{l}{ Proceso de aporque } & \\
\hline Descripción & Medida & Cantidad & Costo Unitario & Importe \\
\hline Peón & Jornal & 6 & $\mathrm{~S} / 30.00$ & $\mathrm{~S} / 180.00$ \\
Comida & Por peón & 6 & $\mathrm{~S} / 5.00$ & $\mathrm{~S} / 30.00$ \\
Chicha & Porongo & 1 & $\mathrm{~S} / 20.00$ & $\mathrm{~S} / 20.00$ \\
Hojas de coca & Libra & 1 & $\mathrm{~S} / 12.00$ & $\mathrm{~S} / 12.00$ \\
Cañazo & Botella & 1 & $\mathrm{~S} / 14.00$ & $\mathrm{~S} / 14.00$ \\
Pago a la cocinera & & 1 & $\mathrm{~S} / 20.00$ & $\mathrm{~S} / 20.00$ \\
\hline TOTAL $(\mathrm{c})$ & & & & $\mathrm{S} / 276.00$ \\
\hline
\end{tabular}

En la tabla 5, se muestra todos los elementos que interviene en el proceso de control de maleza de los terrenos de cultivo de papa nativa, con su respectivo costo unitario.

Tabla 5

Tabulación del costo real del proceso de control de maleza

\begin{tabular}{|c|c|c|c|c|}
\hline 4ta etapa: & Proceso d & l de malez & & \\
\hline Descripción & Medida & Cantidad & Costo Unitario & Importe \\
\hline Peón & Jornal & 6 & $\mathrm{~S} / 30.00$ & $\mathrm{~S} / 180.00$ \\
\hline Comida & Por peón & 6 & $\mathrm{~S} / 5.00$ & $\mathrm{~S} / 30.00$ \\
\hline Chicha & Porongo & 1 & $\mathrm{~S} / 20.00$ & $\mathrm{~S} / 20.00$ \\
\hline Hojas de coca & Libra & 1 & $\mathrm{~S} / 12.00$ & $\mathrm{~S} / 12.00$ \\
\hline Cañazo & Botella & 1 & $\mathrm{~S} / 14.00$ & $\mathrm{~S} / 14.00$ \\
\hline Pago a la cocinera & Jornal & 1 & $\mathrm{~S} / 20.00$ & $\mathrm{~S} / 20.00$ \\
\hline Total (d) & & & & $\mathrm{S} / 277.00$ \\
\hline
\end{tabular}

En la tabla 6, se muestra todos los elementos que interviene en el proceso de cosecha de la papa nativa de la comunidad, con su respectivo costo unitario.

Tabla 6

Tabulación del costo real del proceso de cosecha

\begin{tabular}{lllll}
\hline 5ta etapa: & \multicolumn{4}{l}{ Proceso de Cosecha } \\
\hline Descripción & Medida & Utilizada & Unitario & Importe \\
\hline Peón de recojo & Jornal & 8 & $\mathrm{~S} / 30.00$ & $\mathrm{~S} / 240.00$ \\
Comida & Por peón & 8 & $\mathrm{~S} / 5.00$ & $\mathrm{~S} / 40.00$ \\
Chicha & Porongo & 1 & $\mathrm{~S} / 20.00$ & $\mathrm{~S} / 20.00$ \\
Hojas de coca & Libra & 1 & $\mathrm{~S} / 12.00$ & $\mathrm{~S} / 12.00$ \\
Cañazo & Botella & 2 & $\mathrm{~S} / 28.00$ & $\mathrm{~S} / 28.00$ \\
Pago a la cocinera & Jornal & 1 & $\mathrm{~S} / 20.00$ & $\mathrm{~S} / 20.00$ \\
Costales & Unidad & 25 & $\mathrm{~S} / 1.00$ & $\mathrm{~S} / 25.00$
\end{tabular}




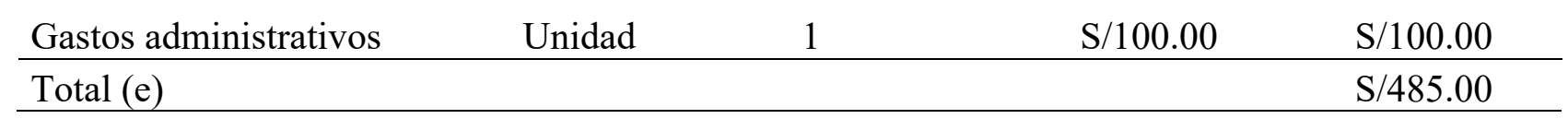

\section{PASO 5: Análisis de costos}

\section{a) Cálculo del costo total de producción}

Para el cálculo total de producción se procedió a sumar todas las etapas de producción.

Costo total de producción $=(a)+(b)+(c)+(d)+(e)$

Costo total de producción $=956.00+758.00+276.00+276.00+485.00$

Costo total de producción $=\mathrm{S} / 2751.00$

Este cálculo indica que, para producir 2500 kilos de papa nativa en la comunidad, a cada productor le cuesta S/ 2751.00 soles.

\section{b) Cálculo del costo de producción unitario o por kilo}

Para obtener el costo de producción se debe considerar que en el área de 0.25 hectáreas, se logró una producción promedio de 2500 kilos, el mismo equivale a 25 sacos de 100 kilos cada uno. Para la obtención de este indicador se aplicó la siguiente fórmula:

Costo de producción por kilo $=\frac{\text { Costo total de producción }}{\text { Cantidad de kilos producidos }}$

Costo de producción por kilo $=\frac{2751 \text { Soles }}{2500 \text { Kilos }}$

Costo de producción por kilo $=1.10$ Soles $/$ Kilo

En este cálculo encontramos que el costo de producción por cada kilo de papa nativa producida es $\mathrm{S} / 1.10$ soles

\section{c) Cálculo del margen bruto de rentabilidad unitario o por kilo}

Para obtener el margen bruto de rentabilidad, se consideró el precio de venta promedio anual, con el que se viene trabajando en la comunidad, el cual equivale a $\mathrm{S} /$ 1.00 cada kilo de papa nativa. Para la obtención de este indicador se aplicó la siguiente formula:

Margen bruto unitario $=$ Precio de venta unitario - Costo de producción unitario

Margen bruto unitario $=1.00-1.10$

Margen bruto unitario $=\mathrm{S} / .-0.10$ por cada kilo 
El margen bruto unitario indica que, por la venta de cada kilo de papa nativa, se está perdiendo 0.10 céntimos de soles generando considerables pérdidas.

\section{d) Cálculo del margen bruto de rentabilidad}

El precio de venta es 1.00 el kilo y la cantidad total de kilos producidos es 2500 kilos en 0.25 hectáreas; el total de ventas generadas serían $\mathrm{S} / 2500.00$ soles, y además se consideró el costo de producción que equivale a S/ 2751.00. Con estos datos se logró determinar el margen bruto de rentabilidad, así como se muestra a continuación:

Margen bruto de rentabilidad $=$ Total de ventas - Costo total de producción

Margen bruto de rentabilidad $=2500-2751.00$

Margen bruto de rentabilidad $=-251.00$

El margen bruto de rentabilidad nos indica que, por la venta de los 2500 kilos de papa nativa, se está perdiendo 251.00 soles, en vez de generar ganancias, cada productor no logra ni recuperar el costo de inversión.

\section{e) Cálculo del porcentaje del margen bruto de rentabilidad}

Para la obtención de este indicador se aplicó la siguiente fórmula:

$\%$ Margen bruto de rentabilidad $=\frac{(\text { Precio de venta unitario }- \text { Costo de producción unitario })}{\text { Precio de venta unitario }}$

$\%$ Margen bruto de rentabilidad $=\frac{1.00-1.10}{1.00}$

$\%$ Margen bruto de rentabilidad $=-10 \%$

El porcentaje de margen bruto de rentabilidad indica que la producción de papa nativa está generando un $10 \%$ de pérdidas económicas a los productores de la comunidad, en vez de generar ganancias económicas.

\section{Resultados y discusiones}

\section{Análisis del costo total de producción}

A continuación, en la tabla 7, se muestra los resultados del costo total de producción de papa nativa en la comunidad de estudio, para lo cual se consideró las 5 etapas del proceso de producción de la misma. 
Tabla 7

Cálculo del costo total de producción de papa nativa

\begin{tabular}{lllll}
\hline Descripción: & Frecuencia & Porcentaje & Acumulado & \% Acumulado \\
\hline Preparación de la tierra & $\mathrm{S} / 956.00$ & $35 \%$ & $\mathrm{~S} / 956.00$ & $34.75 \%$ \\
Proceso de siembra & $\mathrm{S} / 758.00$ & $28 \%$ & $\mathrm{~S} / 1,714.00$ & $62.30 \%$ \\
Proceso de aporque & $\mathrm{S} / 276.00$ & $10 \%$ & $\mathrm{~S} / 1,990.00$ & $72.34 \%$ \\
Proceso de control de maleza & $\mathrm{S} / 276.00$ & $10 \%$ & $\mathrm{~S} / 2,266.00$ & $82.37 \%$ \\
Proceso de Cosecha & $\mathrm{S} / 485.00$ & $18 \%$ & $\mathrm{~S} / 2,751.00$ & $100.00 \%$ \\
\hline Total & $\mathrm{S} / 2,751.00$ & $100 \%$ & & \\
\cline { 1 - 2 } & &
\end{tabular}

En la figura 3, se pueda observar que el proceso de preparación de la tierra y el proceso de siembra representan el porcentaje más influyente, en la elevación del costo de inversión, en la producción de papa nativa, por lo que el gráfico de Pareto sugiere reducir estos costos para optimizar los costos de producción de papa nativa.

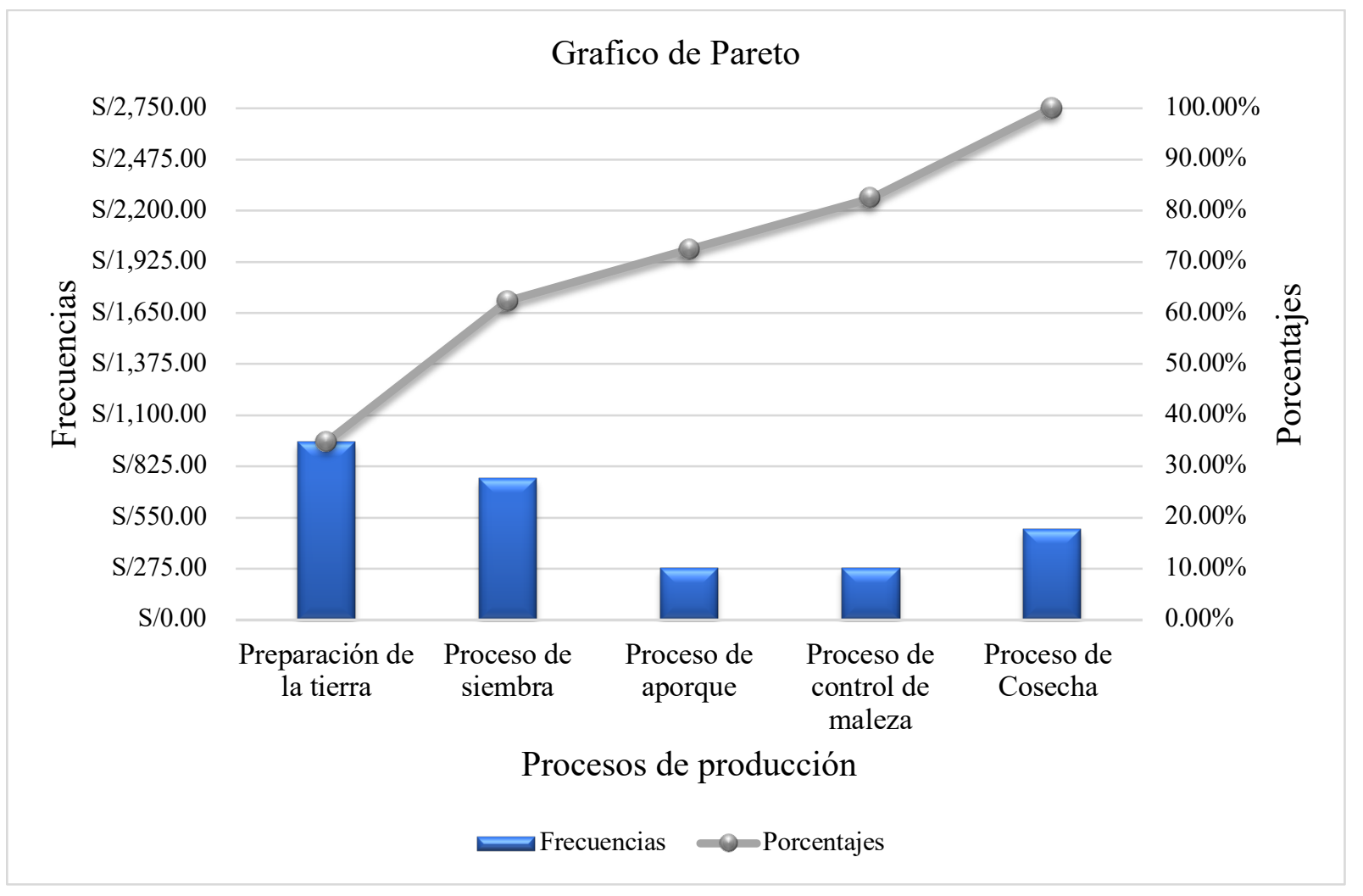

Figura 3. Diagrama de Pareto del costo de producción de papa nativa

\section{Análisis de la rentabilidad}

Como se puede observar en la figura 4, el porcentaje del margen de rentabilidad está por debajo del costo de producción. 


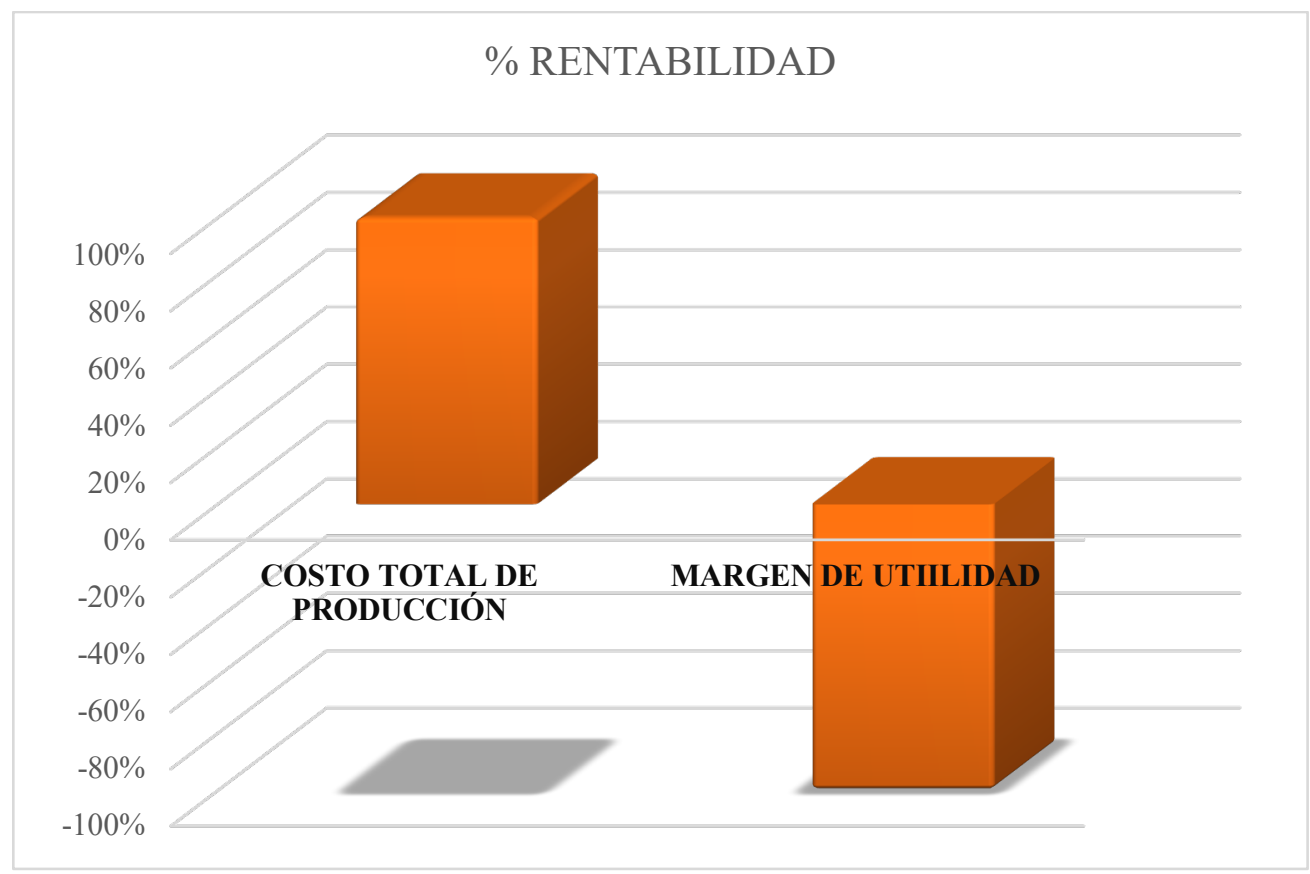

Figura 4. Diagrama de bloques del porcentaje del margen de rentabilidad

Además, en este estudio se determinó el punto de equilibrio a través de la siguiente fórmula:

Punto de equilibrio $=\frac{\text { Costo Fijo }}{\text { Precio de venta unitario-Costo de produccion unitario }}$

En la tabla 8 se puede observar que el punto de equilibrio es negativo, lo cual deja la evidencia de que el negocio no es rentable.

Tabla 8

Cálculo del punto de equilibrio de la producción de papa nativa

\begin{tabular}{lll}
\hline Descripción: & Importe & Unidad \\
\hline Costo Fijo & $\mathrm{S} / 2,751.00$ & Soles \\
Precio de Venta Unitario & $\mathrm{S} / 1.00$ & Soles \\
$\begin{array}{l}\text { Costo de Producción } \\
\text { Unitario }\end{array}$ & $\mathrm{S} / 1.10$ & Soles \\
\hline Punto de equilibrio & -27510 & Kilos a Producir \\
\hline
\end{tabular}

Para la obtención del gráfico del punto de equilibrio, es necesario realizar una proyección de ventas, así como se muestra en la tabla 9. 
Tabla 9

Cálculo del punto de equilibrio de la producción de papa nativa

\begin{tabular}{lllr}
\hline Cantidad & Ventas & Costos totales & Utilidades \\
\hline 1200 & $\mathrm{~S} / 1,200.00$ & $\mathrm{~S} / 4,071.00$ & $-\mathrm{S} / 2,871.00$ \\
3200 & $\mathrm{~S} / 3,200.00$ & $\mathrm{~S} / 6,271.00$ & $-\mathrm{S} / 3,071.00$ \\
5200 & $\mathrm{~S} / 5,200.00$ & $\mathrm{~S} / 8,471.00$ & $-\mathrm{S} / 3,271.00$ \\
7200 & $\mathrm{~S} / 7,200.00$ & $\mathrm{~S} / 10,671.00$ & $-\mathrm{S} / 3,471.00$ \\
9200 & $\mathrm{~S} / 9,200.00$ & $\mathrm{~S} / 12,871.00$ & $-\mathrm{S} / 3,671.00$ \\
11200 & $\mathrm{~S} / 11,200.00$ & $\mathrm{~S} / 15,071.00$ & $-\mathrm{S} / 3,871.00$ \\
13200 & $\mathrm{~S} / 13,200.00$ & $\mathrm{~S} / 17,271.00$ & $-\mathrm{S} / 4,071.00$ \\
15200 & $\mathrm{~S} / 15,200.00$ & $\mathrm{~S} / 19,471.00$ & $-\mathrm{S} / 4,271.00$ \\
17200 & $\mathrm{~S} / 17,200.00$ & $\mathrm{~S} / 21,671.00$ & $-\mathrm{S} / 4,471.00$ \\
19200 & $\mathrm{~S} / 19,200.00$ & $\mathrm{~S} / 23,871.00$ & $-\mathrm{S} / 4,671.00$ \\
21200 & $\mathrm{~S} / 21,200.00$ & $\mathrm{~S} / 26,071.00$ & $-\mathrm{S} / 4,871.00$ \\
23200 & $\mathrm{~S} / 23,200.00$ & $\mathrm{~S} / 28,271.00$ & $-\mathrm{S} / 5,071.00$ \\
25200 & $\mathrm{~S} / 25,200.00$ & $\mathrm{~S} / 30,471.00$ & $-\mathrm{S} / 5,271.00$ \\
\hline
\end{tabular}

Finalmente, en la figura 5 se muestra el gráfico del punto de equilibrio, se puede observar que los valores del punto de equilibrio no llegan a interceptarse, porque se encuentran en valores negativos, indicando que el negocio no llegará a ser rentable, aunque se produzcan cantidades superiores.

\section{Punto de equilibrio}

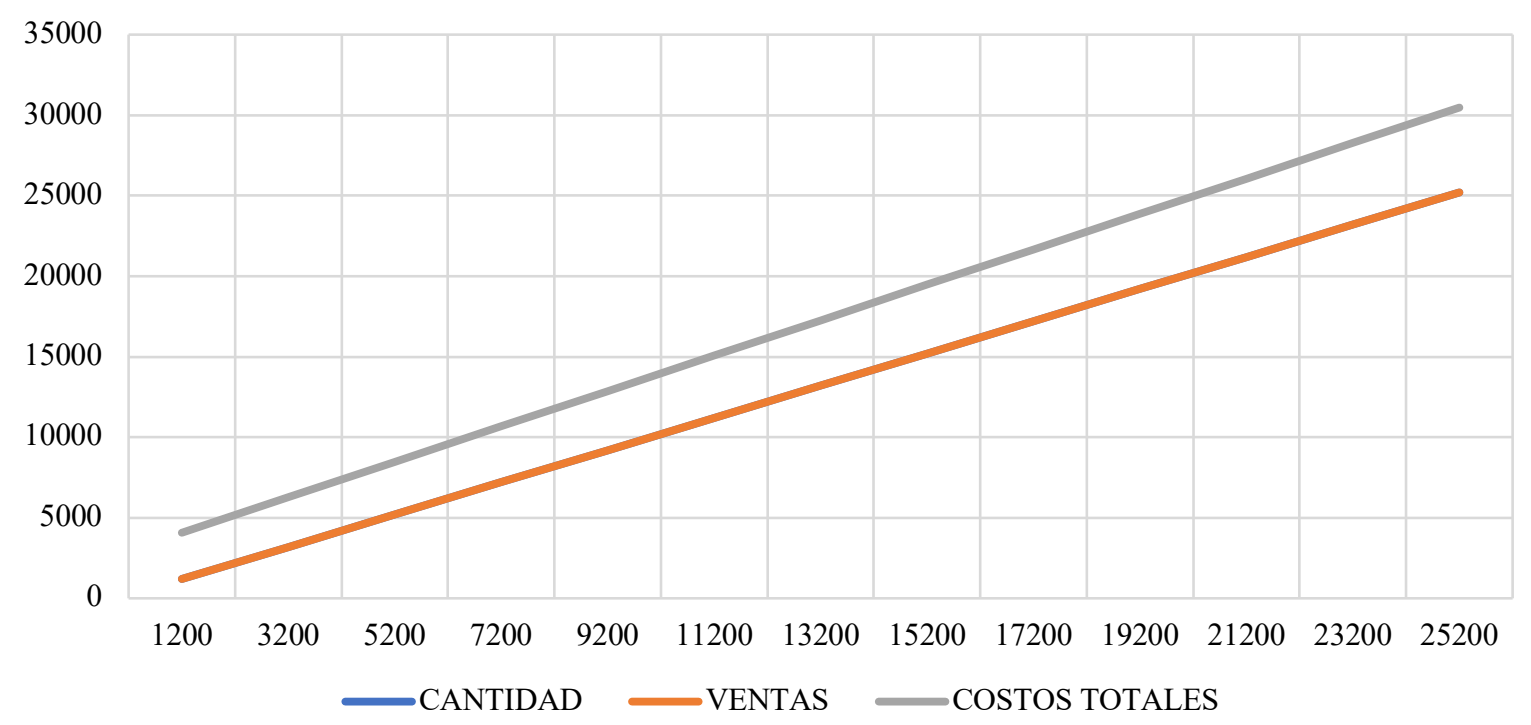

Figura 5. Gráfico del punto de equilibrio de la producción de papa nativa 
En los cálculos de rentabilidad se encontró que la producción de papa nativa de la comunidad no es rentable, indicando un margen de rentabilidad de $-10 \%$; sin embargo, Rodríguez, Pinedo y Sulca (2020) encontraron en su investigación en tres variedades de papa, que un segundo aporque no se justifica en términos económicos; para la variedad Única es suficiente un solo aporque temprano a los 15 días. Además, Estrada (2013) halló un 1,3\% de rentabilidad neta en parcelas de la irrigación Majes-Arequipa. Sin embargo, Chala (2016) informó que los agricultores pueden obtener más ingresos si practican tres aporques, aunque esto incremente los costos en comparación con el aporque en dos oportunidades. Por consiguiente, por razones económicas un solo aporque bien realizado es suficiente. Solo se justifica realizar dos aporques, si las condiciones locales son de alta pluviosidad; en consecuencia de las heladas, algunas partes de interés comercial (tubérculos) pueden resultar afectadas por agentes físicos y biológicos (Egúsquiza, 2014).

\section{Conclusiones}

En este artículo de estudio de costos de la producción de papa nativa, se concluye que el costo de producción por kilo es de $\mathrm{S} / 1.10$ soles; en una producción de 0.25 hectáreas, el costo de producción total es S/ 2751.00 soles. Sin embargo, el precio de venta que la comunidad de Pumapaqcha expende la papa nativa es de $\mathrm{S} / 1.00$, provocando un margen bruto de rentabilidad de $-10 \%$, el mismo que equivale a una pérdida de $\mathrm{S} /$. 275.00 por cada 2500 kilos; a su vez, esto equivale a S/1100.00 soles de pérdida por cada hectárea, dejando la evidencia de la necesidad de optimizar los costos de producción, en los procesos de preparación de la tierra y la siembra de la producción de papa nativa.

\section{Referencias bibliográficas}

Chani, A. y. Pfuro W. (2015). Producción de papas nativas y su industrialización para el mercado de snack del distrito de Cusco. [Tesis, Universidad Nacional de San Antonio

Abad

del

Cusco]. http://repositorio.unsaac.edu.pe/handle/20.500.12918/110

Chala, G. (2016). Effect of earthing up frequencies and tuber seed form on yield and pro tability of potato (Solanum tuberosum) production in Bale highlands. Agricultural Research \& Technology, 2(4), 96-101. http://dx.doi .org/10.19080/ARTOAJ.2016.01.555592. 
Estrada, R. (2013). Momento del aporque en la producción de papa (Solanum tuberosum) cv. "Única" bajo el sistema de riego por goteo en zona árida [Tesis de pregrado, Universidad Nacional de San Agustín de Arequipa]. http://repositorio.unsa.edu.pe/handle/UNSA/4122

Egúsquiza, R. (2014). La papa en el Perú (2nd ed.). Universidad Nacional Agraria La Molina.

FAO, (2003). Organización de Alimentos y Agricultura de las Naciones Unidas [página web]. Recuperado de http://fao.org. (2004, febrero 6).

Joume R, (2013). Teoría y práctica del modelado de procesos mediante Diagramas de Flujo Recuperado de https://www.jramonet.com/sites/default/files/adjuntos/diagramas_flujo_jrf_v201 3.pdf

Hernández, R. y. Mendoza, C. (2018). Metodología de la investigación Las rutas cuantitativa, cualitativa y mixta. México: McGraw Hill Interamericana Editores S.A. de C.V.

Manjarrés, E. (2003). El proceso de cálculo del costo de producción -rubro papa- en cinco sectores del municipio Rangel del estado Mérida (2002). [Trabajo de grado sin publicar. Universidad de Los Andes Mérida Venezuela].

Minaya, C. (2016). Analisis de la rentabilidad en la produccion de papa compis en la regiones de Huanuco y Lima. Anales Cintíficos, 76(2), 369-375, https://doi.org/10.21704/ac.v76i2.803

Monlina de Paredes, O. y Contreras, A (2005). Analisis de los metodos de calculo del costo de produccion de papa en el municipio Rangel del estado Merida año 2005. Visión Gerencial, (1), 103-113, https://www.redalyc.org/pdf/4655/465545880003.pdf

Niño,V. (2011). Metodología de la investigación (1era Edición) Ediciones de la U, Bogotá, Colombia Recuperado de http://roa.ult.edu.cu/bitstream/123456789/3243/1/METODOLOGIA DE LA INVESTIGACION DISENO Y EJECUCION.pdf 
Rodríguez S, Pinedo T., y Sulca S. (2020). Efecto del aporque en el rendimiento y la rentabilidad en cultivares nativos de papa. Ciencia y Tecnología Agropecuaria, 21(3), 1-14, https://doi.org/10.21930/rcta.vol21_num3 art:1798

Segura Salazar, S. (2014). Cadena de valor de papas nativas (solanum andigenum sp) en la provincia de Jauja, Perú. [Tesis doctoral, Universidad Politécnica de Madrid]. https://oa.upm.es/32188/1/BILLY_SEGURA_SALAZAR.pdf

Tamayo, C., y Silva, I. (2010.). Técnicas e instrumentos de recolección de datos. [Tesis, Universidad Católica Los Ángeles de Chimbote]. Recuperado de http://www.postgradoune.edu.pe/pdf/documentos-academicos/ciencias-de-laeducacion/23.pdf 